\title{
THE CLOSE RELATION BETWEEN THE MAXWELL SYSTEM AND THE DIRAC EQUATION WHEN THE ELECTRIC FIELD IS PARALLEL TO THE MAGNETIC FIELD
}

\author{
LA ESTRECHA RELACIÓN ENTRE EL SISTEMA DE MAXWELL Y LA ECUACIÓN \\ DE DIRAC, CUANDO EL CAMPO ELÉCTRICO ES PARALELO AL CAMPO MAGNÉTICO
}

\author{
H. Torres-Silva ${ }^{1}$ \\ Recibido el 5 de septiembre de 2007, aceptado el 5 de diciembre de 2007 \\ Received: September 5, 2007 Accepted: December 5, 2007
}

\begin{abstract}
RESUMEN
En el presente artículo se propone una simple igualdad que considera el operador de Dirac y los operadores de Maxwell bajo un enfoque quiral. Esta igualdad establece una conexión directa entre las soluciones de los dos sistemas. Además se muestra que es válida cuando una relación muy natural se cumple entre la frecuencia de la onda electromagnética y la energía de la partícula Dirac, si el campo eléctrico $\vec{E}$ es paralelo al campo magnético $\vec{H}$. Este análisis se basa en la forma cuaterniónica de la ecuación de Dirac y la forma cuaterniónica de las ecuaciones de Maxwell. En ambos casos las reformulaciones con cuaterniones son completamente equivalentes a la forma tradicional de los sistemas de Dirac y Maxwell. Esta teoría es una nueva interpretación de la mecánica cuántica. Este trabajo prueba que la mecánica cuántica representa la electrodinámica de ondas quirales curvilíneas cerradas. Esto está enteramente de acuerdo con la moderna interpretación y resultados de la teoría cuántica de campo.
\end{abstract}

Palabras clave: Cuaternión, ecuación de Dirac, Sistema de Maxwell.

\begin{abstract}
In the present article we propose a simple equality involving the Dirac operator and the Maxwell operators from a chiral approach. This equality establishes a direct connection between solutions of the two systems. Moreover, we show that the connection is valid when a fairly natural relationship between the frequency of the electromagnetic wave and the energy of the Dirac particle is fulfilled, if the electric field $\vec{E}$ is parallel to the magnetic field $\vec{H}$. Our analysis is based on the quaternionic form of the Dirac equation and on the quaternionic form of the Maxwell equations. In both cases the quaternionic reformulations are completely equivalent to the traditional form of the Dirac and Maxwell systems. This theory is a new quantum mechanics $(Q M)$ interpretation. The research below shows that the QM represents the electrodynamics of the curvilinear closed chiral waves. This concords entirely with the modern interpretation and results of the quantum field theory.
\end{abstract}

Keywords: Quaternion, Dirac equation, Maxwell system.

\section{INTRODUCTION}

The relation between the two most important in mathematical physics first order systems of partial differential equations is among those topics which attract attention because of their general, even philosophical significance but at the same time do not offer much for the solution of particular problems concerning physical models. The Maxwell equations can be represented in a Dirac like form in different ways (e.g., [3, 5, 9]). Solutions of Maxwell's system can be related to solutions of the Dirac equation through some nonlinear equations (e.g., [11]). Nevertheless, in spite of these significant efforts there remain some important conceptual questions.

1 Instituto de Alta Investigación. Universidad de Tarapacá. Antofagasta No 1520. Arica, Chile. E-mail: htorres@uta.cl 
For example, what is the meaning of this close relation between the Maxwell system and the Dirac equation and how this relation is connected with the waveparticle dualism. In the present article we propose a simple equality involving the Dirac operator and the Maxwell operators under chiral approach. This equality establishes a direct connection between solutions of the two systems and moreover, we show that it is valid when a quite natural relation between the frequency of the electromagnetic wave and the energy of the Dirac particle is fulfilled when $\vec{E}$ is parallel to $\vec{H}$. Our analysis is based on the quaternionic form of the Dirac equation obtained in [7] and on the quaternionic form of the Maxwell equations proposed in [6] (see also [8]). In both cases the quaternionic reformulations are completely equivalent to the traditional form of the Dirac and Maxwell systems. Chiral approach means that our Universe is observable area of basic space-time where temporal coordinate is positive and all particles bear positive masses (energies). The mirror Universe is an area of the basic space-time, where from viewpoint of regular observer temporal coordinate is negative and all particles bear negative masses. Also, from viewpoint of our-world observer the mirror Universe is a world with reverse flow of time, where particles travel from future into past in respect to us. The two worlds are separated with the membrane - an area of space-time inhabited by light-like particles that travel along light-like right or left-handed (isotropic-chiral) spirals. On the scales of elementary particles such space can be attributed to particles that possess spirality (e. g. photons). The membrane prevents mixing of positive and negative-mass particles and thus their total annihilation. Exchange interactions between the two worlds can be effected through particles with zero relativistic masses (zeroparticles) under physical conditions that exist on surfaces of collapsers in degenerated spacetime (zero-space).

\section{PRELIMINARIES}

The algebra of complex quaternions is denoted by $H(C)$.

Each complex quaternion $q$ is of the form $q=\sum_{k=0}^{3} q_{k} i_{k}$ where $\left\{q_{k}\right\} \subset C, i_{0}$ is the unit and $\left\{i_{k} \mid k=1,2,3\right\}$ are the quaternionic imaginary units:

$i_{0}^{2}=i_{0}=-i_{k}^{2} ; i_{0} i_{k}=i_{k} i_{0}=i_{k}, k=1,2,3 ;$

$i_{1} i_{2}=-i_{2} i_{1}=i_{3}, i_{2} i_{3}=-i_{3} i_{2}=i_{1}$;

$i_{3} i_{1}=-i_{1} i_{3}=i_{2}$
The complex imaginary unit $i$ commutes with $i_{k}, k=\overline{0,3}$.

We will use the vector representation of complex quaternions: $q=S c(q)+\operatorname{Vec}(q)$, where $S c(q)=q_{0}$ and $\operatorname{Vec}(q)=\vec{q}=\sum_{k=1}^{3} q_{k} i_{k}$. That is each complex quaternion is a sum of its scalar part and its vector part. Complex vectors we identify with complex quaternions whose scalar part is equal to zero. In vector terms, the multiplication of two arbitrary complex quaternions $q$ and $b$ can be written as follows:

$q \cdot b=q_{0} b_{0}-<\vec{q}, \vec{b}>+[\vec{q} \times \vec{b}]+q_{0} \vec{b}+b_{0} \vec{q}$,

where

$$
<\vec{q}, \vec{b}>:=\sum_{k=1}^{3} q_{k} b_{k} \in C
$$

and

$$
[\vec{q} \times \vec{b}]:=\left|\begin{array}{ccc}
i_{1} & i_{2} & i_{3} \\
q_{1} & q_{2} & q_{3} \\
b_{1} & b_{2} & b_{3}
\end{array}\right| \in C^{3}
$$

We shall consider continuously differentiable $H(C)$ -valued functions depending on three real variables $x=\left(x_{1}, x_{2}, x_{3}\right)$. On this set the well known (see, e.g., [1, 4, 7 and 8]) Moisil-Theodoresco operator is defined by the expression

$$
D:=\sum_{k=1}^{3} i_{k} \partial_{k}, \text { where } \partial_{k}=\frac{\partial}{\partial x_{k}}
$$

The action of the operator $D$ on an $H(C)$-valued function $f$ can be written in a vector form:

$$
D f=-\operatorname{div} \vec{f}+\operatorname{grad} f_{0}+\operatorname{rot} \vec{f}
$$

That is, $S c(D f)=-\operatorname{div} \vec{f}$ and $\operatorname{Vec}(D f)=\operatorname{grad} f_{0}+\operatorname{rot} \vec{f}$. In a good number of physical applications (see [4 and 8]) the operators $D_{\alpha}=D+M^{\alpha}$ and $D_{-\alpha}=D-M^{\alpha}$ are needed, where $\alpha$ is a complex quaternion and $M^{\alpha}$ denotes the operator of multiplication by $\alpha$ from the right-hand side: $M^{\alpha} f=f \cdot \alpha$. Here we will be interested in two special cases when $\alpha$ is a scalar, that is $\alpha=\alpha_{0}$ or when $\alpha$ is a vector $\alpha=\vec{\alpha}$. The first case corresponds to the Maxwell equations and the second to the Dirac equation. 


\section{THE DIRAC EQUATION}

Following [7], we consider the Dirac equation in its covariant form

$$
\left(\hbar\left(\frac{\gamma_{0}}{c} \partial_{t}+\sum_{k=1}^{3} \gamma_{k} \partial_{k}\right)+i m c\right) \Phi(t, x)=0 .
$$

For a wave function with a given energy we have $\Phi(t, x)=a(x) e^{i \frac{\varepsilon}{\hbar} t}$, where $\alpha$ satisfies the equation

$$
\left(\frac{i \varepsilon}{c \hbar} \gamma_{0}+\sum_{k=1}^{3} \gamma_{k} \partial_{k}+\frac{i m c}{\hbar}\right) a(x)=0
$$

Denote

$$
D:=\frac{i \varepsilon}{c \hbar} \gamma_{0}+\sum_{k=1}^{3} \gamma_{k} \partial_{k}+\frac{i m c}{\hbar}
$$

Let us introduce an auxiliary notation $\tilde{f}:=f\left(t, x_{1}, x_{2},-x_{3}\right)$. The transformation which allows us to rewrite the Dirac equation in a quaternionic form we denote as $A$ and define in the following way [7]. A function $\Phi: R^{3} \rightarrow C^{4}$ is transformed into a function $F: R^{3} \rightarrow H(C)$ by the rule

$$
F=\mathcal{A}[\Phi]=\frac{1}{2}\left(-\left(\tilde{\Phi}_{1}-\tilde{\Phi}_{2}\right) i_{0}+i\left(\tilde{\Phi}_{0}-\tilde{\Phi}_{3}\right) i_{1}-\left(\tilde{\Phi}_{0}+\tilde{\Phi}_{3}\right) i_{2}+i\left(\tilde{\Phi}_{1}+\tilde{\Phi}_{2}\right) i_{3}\right)
$$

The inverse transformation $A^{-1}$ is defined as follows

$$
\Phi=\mathcal{A}^{-1}[F]=\left(-i \tilde{F}_{1}-\tilde{F}_{2},-\tilde{F}_{0}-i \tilde{F}_{3}, \tilde{F}_{0}-i \tilde{F}_{3}, i \tilde{F}_{1}-\tilde{F}_{2}\right)
$$

Let us present the introduced transformations in a more explicit matrix form which relates the components of a $\mathrm{C}^{4}$-valued function $\Phi$ with the components of an $H(C)$ -valued function $F$ :

$$
F=\mathcal{A}[\Phi]=\frac{1}{2}\left(\begin{array}{cccc}
0 & -1 & 1 & 0 \\
i & 0 & 0 & -i \\
-1 & 0 & 0 & -1 \\
0 & i & i & 0
\end{array}\right)\left(\begin{array}{c}
\tilde{\Phi}_{0} \\
\tilde{\Phi}_{1} \\
\tilde{\Phi}_{2} \\
\tilde{\Phi}_{3}
\end{array}\right)
$$

and

$$
\Phi=\mathcal{A}^{-1}[F]=\left(\begin{array}{cccc}
0 & -i & -1 & 0 \\
-1 & 0 & 0 & -i \\
1 & 0 & 0 & -i \\
0 & i & -1 & 0
\end{array}\right)\left(\begin{array}{l}
\tilde{F}_{0} \\
\tilde{F}_{1} \\
\tilde{F}_{2} \\
\tilde{F}_{3}
\end{array}\right) .
$$

We have the following important equality

$$
D_{\vec{\alpha}}=-\mathcal{A} \gamma_{1} \gamma_{2} \gamma_{3} D \mathcal{A}^{-1},
$$

where $\vec{\alpha}:=-\frac{1}{\hbar}\left(i \frac{\varepsilon}{c} i_{1}+m c i_{2}\right)$. This equality shows us that instead of equation (2) we can consider the equation

$$
D_{\vec{\alpha}} f=0
$$

and the relation between solutions of (2) and (4) is established by means of the invertible transformation $\mathcal{A}: f=\mathcal{A} q$.

\section{THE CHIRAL MAXWELL EQUATIONS}

We will consider the time-harmonic Maxwell equations for a sourceless isotropic chiral homogeneous medium with: $\vec{E}=\varepsilon^{-1}(\vec{D}+T \nabla \times \vec{D})$ and $\vec{B}=\mu\left(\vec{H}^{*}+T \nabla \times \vec{H}^{*}\right)$ [12], so $\vec{H}=\vec{H}^{*}+T \nabla \times \vec{H}^{*}$. Then we have

$$
\begin{gathered}
\operatorname{rot} \vec{H}=-i \omega \varepsilon \vec{E}, \\
\operatorname{rot} \vec{E}=i \omega \mu \vec{H}, \\
\operatorname{div} \vec{E}=0, \\
\operatorname{div} \vec{H}=0 .
\end{gathered}
$$

Here $T$ is the chiral scalar parameter, $\omega$ is the frequency, $\varepsilon$ and $\mu$ are the absolute permittivity and permeability respectively. $\varepsilon=\varepsilon_{0} \varepsilon_{r}$ and $\mu=\mu_{0} \mu_{r}$, where $\varepsilon_{0}$ and $\mu_{0}$ are the corresponding parameters of a vacuum and $\varepsilon_{r}, \mu_{r}$ are the relative permittivity and permeability of a medium.

Taking into account (1) we can rewrite this system as follows

$$
D \vec{E}=i \omega \mu \vec{H},
$$




$$
D \vec{H}=-i \omega \varepsilon \vec{E}
$$

This pair of equations can be diagonalized in the following way [6] (see also [8]). Denote

$$
\vec{\varphi}:=-i \omega \varepsilon \vec{E}+\kappa \vec{H}
$$

and

$$
\vec{\psi}:=i \omega \varepsilon \vec{E}+\kappa \vec{H}
$$

Where $\kappa:=\omega \sqrt{\varepsilon \mu}=\frac{\omega}{c} \sqrt{\varepsilon_{r} \mu_{r}}$ is the wave number. Applying the operator $D$ to the functions $\vec{\varphi}$ and $\vec{\psi}$ one can see that $\vec{\varphi}$ satisfies the equation

$$
(D-\kappa) \vec{\varphi}=0
$$

and $\vec{\psi}$ satisfies the equation

$$
(D+\kappa) \vec{\psi}=0 .
$$

Solutions of (13) and (14) are called the Beltrami fields (see, e.g., [10]).

\section{THE RELATION $\omega(\kappa)$ WHEN $\vec{E}$ IS PARALLEL TO $\vec{H}$}

In the preceding sections it was shown that the Dirac equation (2) is equivalent to the equation $D_{\vec{\alpha}} f=0$ with $\vec{\alpha}:=-\frac{1}{\hbar}\left(i \frac{\varepsilon}{c} i_{1}+m c i_{2}\right)$ and the Maxwell equations (5)-(8) are equivalent to the pair of quaternionic equations $D_{-\kappa} \vec{\varphi}=0$ and $D_{\kappa} \vec{\psi}=0$. Now we will show a simple relation between these objects. Suppose that

$$
\frac{1}{T^{2}}=\kappa^{2}=\vec{\alpha}^{2}=\frac{\omega^{2}}{c^{2}} .
$$

Let us introduce the following operators of multiplication

$$
P^{ \pm}:=\frac{1}{2 \kappa} M^{\kappa \pm \vec{\alpha}}
$$

It is easy to verify that they are mutually complementary and orthogonal projection operators, and the following equality is valid [8]

$$
D_{\vec{\alpha}}=P^{+} D_{\kappa}+P^{-} D_{-\kappa} .
$$

Moreover, as $P^{ \pm}$commutes with $\mathrm{D}_{ \pm \kappa}$, we obtain that any solution of (4) is uniquely represented as follows

$$
f=P^{+} \psi+P^{-} \varphi
$$

Where $\varphi$ and $\psi$ are solutions of (13) and (14) respectively but in general can be full quaternions not necessarily purely vectorial. In particular, we have that

$$
\begin{aligned}
f & =P^{+}(i \omega \varepsilon \vec{E}+\kappa \vec{H})+P^{-}(-i \omega \varepsilon \vec{E}+\kappa \vec{H}) \\
& =i \omega \varepsilon\left(P^{+}-P^{-}\right) \vec{E}+\kappa\left(P^{+}+P^{-}\right) \vec{H} \\
& =\frac{i \omega \varepsilon}{\kappa} \vec{E} \cdot \vec{\alpha}+\kappa \vec{H}
\end{aligned}
$$

is a solution of (4) if $\vec{E}$ and $\vec{H}$ are solutions of (5)-(8). It should be noticed that (16) works in both directions. We have

$$
D_{\kappa}=P^{+} D_{\vec{\alpha}}+P^{-} D_{-\vec{\alpha}}
$$

and

$$
D_{-\kappa}=P^{+} D_{\vec{\alpha}}+P^{-} D_{-\vec{\alpha}}
$$

The fact that the Maxwell system reduces to equations (13) and (14), where the functions $\vec{\varphi}$ and $\vec{\psi}$ are purely vectorial provokes the natural question whether it had any sense to consider full quaternions $\vec{\varphi}$ and $\psi$ and hence four-component vectors $E$ and $H$ or the nature definitely eliminated their scalar parts. Some arguments supporting the idea of nonzero scalar parts can be found, for example, in [2].

As we have seen equality (16) is valid under the condition (15). Let us analyze this condition. Note that

$$
\vec{\alpha}^{2}=-<\vec{\alpha}, \vec{\alpha}>=\frac{1}{\hbar^{2}}\left(\frac{\varepsilon^{2}}{c^{2}}-m^{2} c^{2}\right) .
$$

Thus, when $\vec{E}$ is parallel to $\vec{H},(15)$ has the form

$$
\frac{1}{T^{2}}=\kappa^{2}=\frac{1}{\hbar^{2}}\left(\frac{\varepsilon^{2}}{c^{2}}-m^{2} c^{2}\right)
$$


or equivalently

$$
(\hbar \omega)^{2} \varepsilon_{r} \mu_{r}=\varepsilon^{2}-m^{2} c^{4}
$$

From this equation in the case $\varepsilon_{r}=\mu_{r}=1$, that is for a vacuum, using the well known in quantum mechanics relation between the frequency and the impulse: $\hbar \omega=p c$ we obtain the equality

$$
\varepsilon^{2}=p^{2} c^{2}+m^{2} c^{4}
$$

In general, if in (17) we formally use the de Broglie equality $p=\hbar \kappa=\hbar / T$, we again obtain the fundamental relation (18).

Thus relation (15) between the Dirac operator and the Maxwell operators is valid if the condition (17) is fulfilled which quite is in agreement with (18), if and only if $\vec{E}$ is parallel to $\vec{H}$.

\section{CONCLUSIONS}

The main result of this paper is that the Dirac equation can be derived from the Maxwell's equation under a chiral approach (equation 15). The suggested theory is the new quantum mechanics $(\mathrm{QM})$ interpretation.

The below research proves that the QM represents the electrodynamics of the curvilinear closed (non-linear) waves. It is entirely according to the modern interpretation and explains the particularities and the results of the quantum field theory.

Chiral approach means that our Universe is observable area of basic space-time where temporal coordinate is positive and all particles bear positive masses (electrons). The mirror Universe is an area of the basic space-time, where from viewpoint of regular observer temporal coordinate is negative and all particles bear negative masses (positrons). Also, from viewpoint of our-world observer the mirror Universe is a world with reverse flow of time, where particles travel from future into past in respect to us. The two worlds are separated with the membrane - an area of space-time inhabited by light-like particles that travel along light-like right or left-handed (isotropic) spirals (chiral photons).
[1] F. Brackx, R. Delanghe and F. Sommen. Clifford analysis. Pitman Res. Notes in Math. 1982.

\section{REFERENCES}

[2] K. Carmody. Circular and hyperbolic quaternions, octonions, and sedenions-further results. Applied Mathematics and Computation. Vol. $84 \mathrm{~N}^{\mathrm{o}} 1$, pp. 27-47. 1997.

[3] W. Greiner. "Relativistic quantum mechanics". Springer-Verlag. 1990.

[4] K. Gürlebeck and W. Sprößig. "Quaternionic analysis and elliptic boundary value problems". Akademie-Verlag. 1989.

[5] K. Imaeda. "A new formulation of classical electrodynamics". Nuovo Cimento. Vol. 32 B N $^{\circ} 1$, pp. 138-162. 1976.

[6] M. Gogberashvili. "Octonionic version of Dirac equations". International Journal of Modern Physics A. Vol. 21 No $^{\mathrm{1}}$ 17, pp. 3513-3523. 2006.

[7] V.V. Kravchenko. "On a biquaternionic bag model". Zeitschrift für Analysis und ihre Anwendungen. Vol. $14 \mathrm{~N}^{\circ}$ 1, pp. 3-14. 1995.

[8] V.V. Kravchenko and M.V. Shapiro. "Integral representations for spatial models of mathematical physics". Addison Wesley Longman Ltd., Pitman Res. Notes in Math. Series. Vol. 351. 1996.

[9] I. Yu. Krivsky, V.M. Simulik. "Unitary connection in Maxwell-Dirac isomorphism and the Clifford algebra". Advances in Applied Clifford Algebras. Vol. $6 \mathrm{~N}^{\circ} 2$ 2, pp. 249-259. 1996.

[10] A. Lakhtakia. Beltrami fields in chiral media. World Scientific. 1994.

[11] J. Vaz, Jr., W. Rodrigues, Jr. "Equivalence of Dirac and Maxwell equations and quantum mechanics". International Journal of Theoretical Physics. Vol. 32 No 6, pp. 945-959. 1993.

[12] H. Torres-Silva and M. Zamorano Lucero. Chiral Electrodynamic. URLs: http://www.chiral.cl 